\title{
Relaciones filogenéticas entre las especies del género Cynoscionicola (Monogenea: Microcotylidae)
}

\author{
Maria Berenit Mendoza Garfias ${ }^{1}$ y Gerardo Pérez-Ponce de León ${ }^{1}$ \\ 1 Laboratorio de Helmintología, Instituto de Biología UNAM, Ap. Postal 70-153. C.P. 04510 México, D.F., email: \\ berenit@ ibiologia.unam.mx; ppdleon@servidor.unam.mx
}

Recibido 18-IV-1997. Corregido 8-XII-1997. Aceptado 14-I-1998.

\begin{abstract}
Phylogenetic analysis of seven nominal species of the genus Cynoscionicola Price 1962 based on 18 characteres and 31 character states, produced two equally parsimonious cladograms, with a consistency index of 0.85 and 35 steps. Different tree topologies depends on the position of the members that inhabit scianids in the Pacific Coast, with respect to the ones that inhabit Cynoscion in the Atlantic. Monophyly of Cynoscionicola is supported by three unambiguos synapomorphies: genital atrium formed by four atrial pouches, genital atrium presenting spines of different size and shape, and shape of spines in anterior chamber of atrial pouch. Using phylogenetic analysis as a template, we analyzed host relationships, and biogeographic distribution for members of Cynoscionicola, and we propose an origin for both, hosts and monogeans, in the Atlantic, with an early dispersal route along the Atlantic Coast, and a speciation event (vicariance) determined by the isolation of a population of Cynoscion when the Ithsmus of Panama arose in the Pliocen, combined with a host switching event to other members of the Scianidae.
\end{abstract}

Key words: Phylogeny, phylogenetic analysis, parasites, Microcotylidae, Monogenea, Cynoscionicola, coevolution, biogeography.

El cladismo o sistemática filogenética es una de las escuelas en taxonomía contemporánea que ha discutido y propuesto que la historia evolutiva de un grupo puede ser determinada mediante los caracteres que definen a un grupo monofilético. Según esta lógica, solamente los caracteres derivados compartidos o sinapomorfías proporcionan evidencias de un origen común; con base en estas consideraciones es que pueden establecerse grupos monofiléticos. Una sinapomorfía representa un cambio evolutivo y su origen puede ser explicado mediante una hipótesis de genealogía; en contraste, los caracteres ancestrales (plesiomorfías) y los ancestrales compartidos (simplesio- morfías) no son útiles para determinar grupos monofiléticos pues no son caracteres directamente heredados de un ancestro común inmediato (Wiley 1981).

Los monogéneos representan un excelente sistema de estudio para analizar aspectos de coevolución y biogeografía en un contexto filogenético, en virtud de que presentan ciclos de vida directos y exhiben patrones muy marcados de especificidad hospedatoria. El género Cynoscionicola, incluye en la actualidad siete especies: C. heteracantha (Manter 1938) Price 1962, C. pseudoheteracantha (Hargis 1957) Price 1962, C. srivastavai Bravo \& Caballero 
1970, C. sciaenae Tantalean 1974, C. similis Lambert \& Euzet 1979, C. jamaicensis Lambert \& Euzet 1979 y C. powersi Payne 1991; todos ellos son parásitos exclusivamente de peces de la familia Sciaenidae y tienen una distribución geográfica que se restringe a los litorales occidental y oriental del Continente Americano y cuyo carácter morfológico diagnóstico, es la presencia de un atrio genital complejo provisto de cuatro bolsas atriales de consistencia muscular, con un número variable de espinas de diferente forma y tamaño (Lambert \& Euzet 1979). El objetivo de este trabajo es inferir las relaciones filogenéticas entre las especies del género Cynoscionicola y con base en la hipótesis generada, discutir los procesos que determinan el patrón coevolutivo y biogeográfico observado.

\section{MATERIALES Y MÉTODOS}

Táxones reconocidos: Se realizó un análisis sistemático del género Cynoscionicola (Cuadro 1) para lo cual se recopiló la información bibliográfica disponible sobre los representantes de la subfamilia Anchoromicrocotylinae y se estudiaron los siguientes ejemplares, depositados en tres colecciones: la Colección Nacional de Helmintos, Instituto de Biología, UNAM, México (CNHE): Anchoromicrocotyle guaymensis Bravo 1981 (No. de Cat. 000191, 000192), C. pseudoheteracantha (000015), C. srivastavai (000007, 000004, 2879, 2880, 2881) (Fig. 1), C. sciaenae $(000277,000278)$ y C. jamaicensis (000326); la U.S. National Parasite Collection (USNPC), Beltsville, Maryland: Microcotyle sp (No. Cat. 8166), M. heteracantha (9191), C. heteracantha (37743) y M. pseudoheteracantha (38251); Harold W. Manter Laboratory (UNSMHWML), Division of Parasitology, University of Nebraska State Museum, Lincoln: C. powersi $(31153,31155$, 31156). La validez taxonómica de $C$. sciaenae ha sido puesta en duda en virtud de que exhibe una gran semejanza con $C$. srivastavai, esto, asociado con la imposibilidad de estudiar el material tipo ha determinado que sea conside-

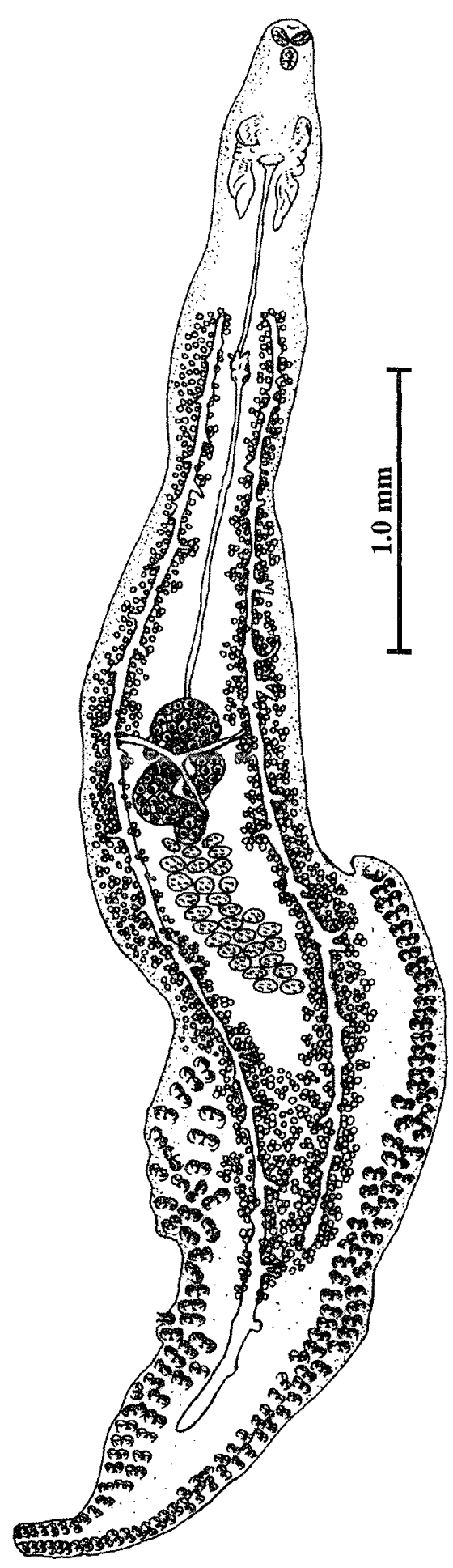

Fig. 1. Cynoscionicola srivastavai. Vista ventral. 
rada como species inquerendae por Jiménez (1996). En este estudio se consideró a C. sciaenae como válida en función de que difiere de $C$. srivastavai en el número y forma de las espinas atriales anterolaterales, en el número de espinas de las bolsas atriales posteriores y en el número de testículos. Finalmente, dado que no fue posible obtener ni las descripciones originales, ni los ejemplares de las especies: $C$. americana Tantaleán, Martínez \& Escalante 1987, C. cynoscioni Tantaleán, Martínez \& Escalante 1987 y $C$. intermedia Tantaleán, Escalante \& Martínez 1988, estas fueron consideradas como species inquerendae.

Análisis realizados: La hipótesis inicial de las relaciones entre las especies del género Cynoscionicola, fue construida utilizando la ar- gumentación de Hennig, los resultados fueron confirmados utilizando el programa de computación PAUP (Phylogenetic Analysis Using Parsimony, versión 3.1.1) (Swofford 1993).

Un total de 18 caracteres con 31 estados de carácter (Cuadro 2) fueron usados en el análisis (13 binarios y 5 multiestado). La polarización de los caracteres se determinó con base en el método del grupo externo (Watrous \& Wheeler 1981, Maddison et al. 1984), estableciendo así el estado plesiomórfico y apomórfico para cada uno de ellos. El grupo externo primario estuvo representado por Anchoromicrocotyle guaymensis, grupo cercanamente $\mathrm{r}$ lacionado con el género Cynoscionicola, de acuerdo con Mamaev (1986); el grupo externo alternativo estuvo representado por Microcotyle donavini

\section{CUADRO 1}

Composición específica del género Cynoscionicola

Especie Hospedero

C. heteracantha

C. jamaicensis

C. pseudoheteracantha

C. sciaenae

C. similis

C. srivastavai

C. powersi
Localidad

Cynoscion nebulosus

Cynoscion regalis

Eriscion nebulosus (sin.

Cynoscion nebulosus)

Cynoscion acoupa

Cynoscion nothus

"Silver seatrout"

Sciaena deliciosa

Umbrina xanti

Cynoscion similis

Bairdiella icistia

Cynoscion xanthulus Isopisthus altipinni

Umbrina roncador

Umbrina sinaloae

Umbrina xanti

Menticirrhus undulatus

Seriphus politus

Umbrina roncador
Beaufort, Carolina del Norte, EEUU., Bahía Chesapeake, Carolina del Norte EEUU., Alligator Harbor, Florida. EEUU., Bahía Chesapeake. EEUU., Woods Hole, Masachusets, EEUU. Beaufort, Carolina del Norte, EEUU.

Carolina del Norte, EEUU.

Guyana Rio de Janeiro, Brasil.

Gran Isla, Jefferson, Parish La. EEUU., Luisiana, EEUU., Jicacal, Veracruz, Méx..

Bahía Chesapeake, EEUU.

Callao y Chorrillos, Perú.

Guaymas, Sonora, Méx.

Guyana

Guaymas, Sonora., Bahía de Matanchen, San Blas, Nayarit, Méx. Guaymas, Sonora, Méx.

Isla de Enfrente, Mazatlán, Sinaloa y Puerto Peñasco, Sonora, Méx. Salina Cruz, Oaxaca, Méx.

La Paz, Baja California Sur, Méx.

Chamela, Jalisco, Méx.

La Jolla, California, EEUU. 
Beneden \& Hesse 1863, especie tipo de ese género. Los caracteres del cinco al 14, fueron polarizados con respecto al grupo externo alternativo, dado que en éste se encontró la condición plesiomórfica para dichos caracteres. Se examinaron las siguientes opciones: los caracteres multiestado desordenados. Optimización: Acctran. Construcción de cladogramas; búsqueda exhaustiva.

Argumentación de caracteres: A continuación se presenta la argumentación de los caracteres utilizados en el análisis filogenético (Cuadro 2); 0 representa el estado plesiomórfico.

1. Lengüeta larval: La presencia de esta estructura en el estado juvenil y/o adulto, es la condición plesiomórfica (0), mientras que su ausencia es el estado apomórfico (1).

2. Pene: Esta estructura se encuentra presente en el género Achoromicrocotyle, por lo que su presencia se considera como el estado plesiomórfico (0) y la presencia de este órgano es el estado apomórfico (1).

3. Naturaleza del atrio genital: $\mathrm{Si}$ el atrio genital no está armado, es el estado plesiomórfico (0), mientras que la presencia de espinas, es el estado apomórfico (1).
4. Forma del atrio genital: (Fig. 2) Este es un carácter multiestado: poseer dos bolsas atriales de naturaleza glandular dispuestas paralelamente, es la condición plesiomórfica (0); presentar dos cámaras o bolsas atriales de naturaleza muscular dispuestas longitudinalmente (1); o bien, presentar cuatro bolsas atriales de naturaleza muscular (2), son las condiciones derivadas.

5. Espinas del atrio genital: Espinas con la misma forma y tamaño se encuentran presentes en Microcotyle, por lo que es la condición plesiomórfica (0), mientras que espinas con diferente forma y tamaño, representan la condición apomórfica (1).

6. Forma de las espinas de la región anterior del atrio genital: (Fig. 3a). La presencia de espinas con una hoja simple sin guarda, es la condición plesiomórfica (0), el presentar espinas con hoja simple, guarda y mango profundas, es la condición apomórfica (1).

7. Número de las espinas de la región anterior del atrio genital: El presentar más de 21 espinas, es la condición plesiomórfica (0), mientras que presentar menos de 18 espinas es la apomórfica (1).

\section{CUADRO 2}

Matriz de datos para las especies del género Cynoscionicola. Los caracteres se ordenan de acuerdo con la numeración del texto; la descripción de los caracteres y los argumentos de polaridad se detallan en el texto. 0 = estado plesiomórfico; $1-5=$ estado apomórfico; ? = datos faltantes

$\begin{array}{lllllllllllllllllll} & 1 & 2 & 3 & 4 & 5 & 6 & 7 & 8 & 9 & 10 & 11 & 12 & 13 & 14 & 15 & 16 & 17 & 18 \\ \text { Mdon } & 0 & 1 & 1 & 1 & 0 & 0 & 0 & 0 & 0 & 0 & 0 & 0 & 0 & 0 & 0 & 0 & 0 & 0 \\ \text { Aguy } & 0 & 0 & 0 & 0 & 0 & 0 & 0 & 0 & 0 & 0 & 0 & 0 & 0 & 0 & 0 & 0 & 0 & 1 \\ \text { Chet } & 1 & 1 & 1 & 2 & 1 & 1 & 0 & 1 & 3 & 0 & 2 & 4 & 0 & 1 & 0 & 1 & 0 & 0 \\ \text { Cpse } & 1 & 1 & 1 & 2 & 1 & 1 & 1 & 0 & 1 & 1 & 1 & 3 & 0 & 1 & 0 & 0 & 1 & 0 \\ \text { Csri } & 1 & 1 & 1 & 2 & 1 & 1 & 1 & 0 & 5 & 1 & 3 & 2 & 1 & 0 & 0 & 0 & 1 & 0 \\ \text { Csci } & 1 & 1 & 1 & 2 & 1 & 1 & 0 & 1 & 2 & 1 & 2 & 2 & 1 & 1 & 0 & 0 & 1 & 0 \\ \text { Csim } & 1 & 1 & 1 & 2 & 1 & 1 & 1 & 1 & 4 & 1 & 1 & 1 & 0 & 1 & 1 & 0 & ? & 0 \\ \text { Cjam } & 0 & 1 & 1 & 2 & 1 & 1 & 1 & 2 & 1 & 1 & 1 & 5 & 0 & 1 & 1 & 0 & 1 & 0 \\ \text { Cpow } & 1 & 1 & 1 & 2 & 1 & 1 & 0 & 1 & 5 & 1 & 3 & 2 & 1 & 1 & 0 & 0 & 1 & 0\end{array}$

Mdon = Microcotyle donavini $;$ Aguy = Achoromicrocotyle guaymensis $;$ Chet = Cynoscionicola heteracantha $;$ Cpse =C.pseudoheteracantha; Csri $=C$ srivastavai $; \mathrm{Csci}=C$. sciaenae $; \mathrm{Csim}=C$. similis $; \mathrm{Cjam}=C$. jamaicensis; $\mathrm{Cpow}=C$. powersi . 

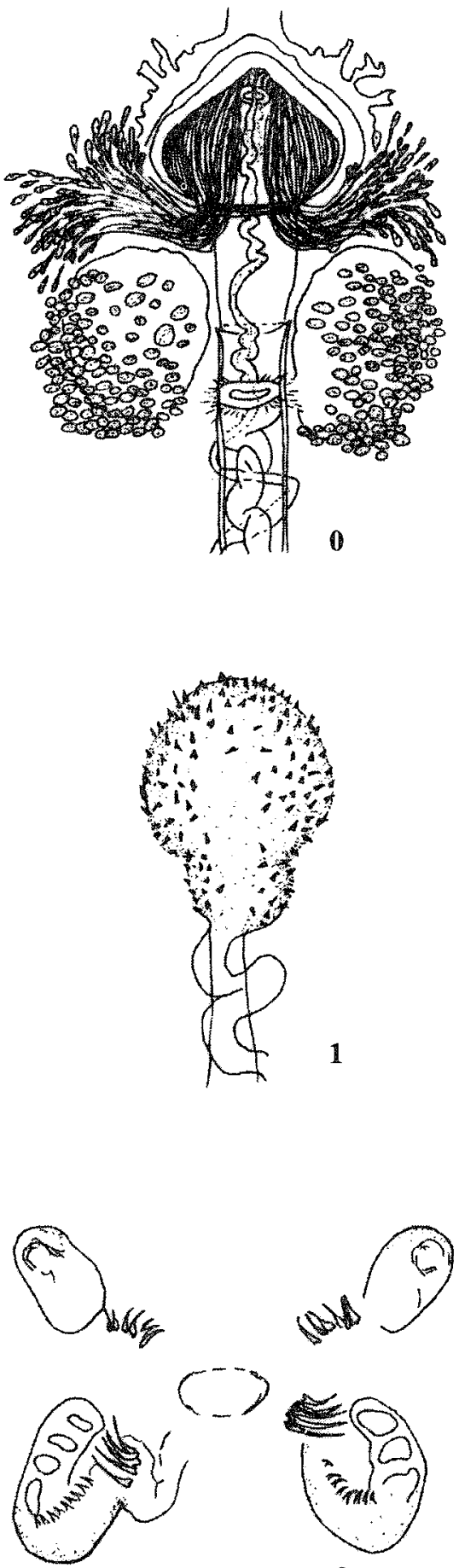

2

Fig. 2. Estados de caracter para la forma del atrio genital. (0) condición plesiomórfica; (1), (2) condición apomórfica (ver argumentación de caracteres).
8. Tamaño de las espinas de la región anterior del atrio genital: (Fig. 3a). Este carácter es multiestado: El estado plesiomórfico (0), se presenta cuando las espinas son menores a $0.009 \mathrm{~mm}$, cuando las espinas son mayores a $0.01 \mathrm{~mm}$ pero menores a $0.019 \mathrm{~mm}$ es un estado apomórfico (1), o bien, si su tamaño es mayor a $0.02 \mathrm{~mm}$ (2), representan otro estado apomórfico.

9. Forma de las espinas de la región anterolateral del atrio genital: (Fig. 3b). Este es un carácter multiestado: la condición plesiomórfica (0), se presenta cuando las espinas poseen una hoja simple sin guarda y mango evidentes; la condición derivada se presenta cuando las espinas poseen una hoja simple con guarda y mango profundos (1); si tienen una hoja simple, guarda y mango del mismo tamaño y en la misma posición (2); espinas con hoja simple con el mango y la guarda alargados y de forma triangular (3); espinas con hoja trífida (4); combinación de espinas con hoja simple y espinas con hoja bífida (5).

10. Número de las espinas de la región anterolateral del atrio genital: El presentar más de diez espinas, es la condición plesiomórfica (0), mientras que presentar menos de nueve (1), es la apomórfica.

11. Tamaño de las espinas de la región anterolateral del atrio genital: (Fig. 3b). Este carácter es multiestado: El estado plesiomórfico (0), se presenta cuando las espinas son pequeñas menores a $0.009 \mathrm{~mm}$, si las espinas son grandes, mayores a $0.02 \mathrm{~mm}$, pero menores a $0.035 \mathrm{~mm}$ el estado es (1), si presenta espinas mayores a $0.036 \mathrm{~mm}$ el estado es (2), si presentan espinas pequeñas y grandes, el estado es (3).

12. Forma de las espinas de la región posterior del atrio genital: (Fig. 3c). Este es un carácter multiestado: Cuando las espinas 
a

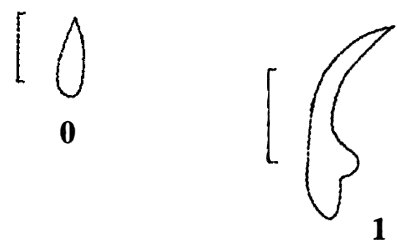

b

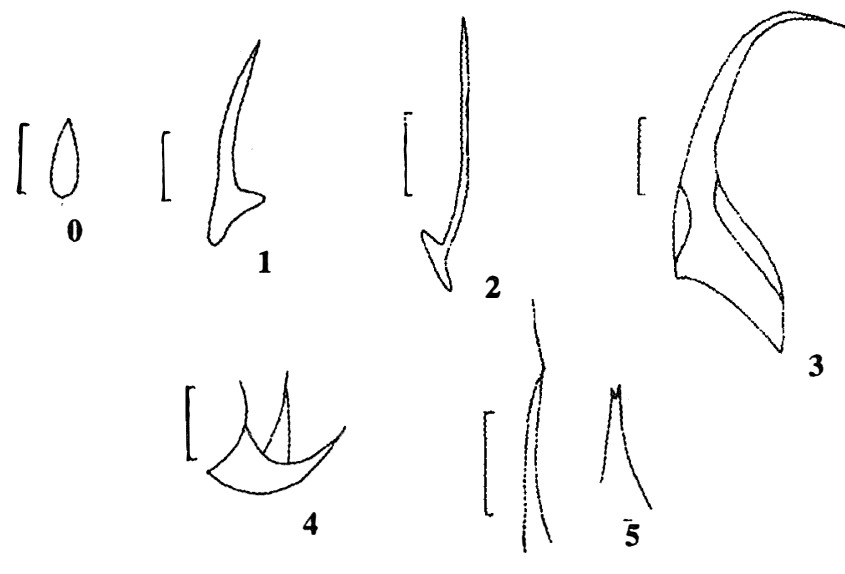

c

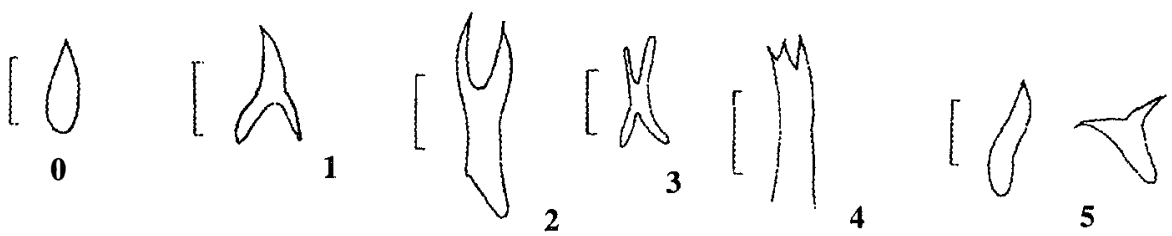

Fig. 3. Estados de caracter para la forma y tamaño de las espinas del atrio genital: a) anteriores, b) anterolaterales, c) posteriores, (0) es la condición plesiomórfica y 1-5 la apomórfica (ver argumentación de caracteres). La escala indicada en cada espina corresponde a $0.01 \mathrm{~mm}$.

presentan una hoja simple sin guarda y mango evidentes, la condición es plesiomórfica (0); las condiciónes derivadas son: presentar espinas con hoja simple, guarda y mango profundo (1), espinas con la hoja bífida sin guarda y mango (2); espinas con hoja bífida con guarda y mango evidentes (3); espinas con la hoja trífida (4) y (5) si hay tanto espinas con hoja simple, como espinas con hoja bífida. 
13. Número de las espinas de la región posterior del atrio genital: El presentar menos de 35 espinas, es la condición plesiomórfica (0), mientras que presentar más de 40 espinas es la condición apomórfica (1).

14. Tamaño de las espinas de la región posterior del atrio genital: (Fig. 3c). El estado plesiomórfico (0), se presenta cuando las espinas son pequeñas (menores a 0.009 $\mathrm{mm}$ ), si las espinas son grandes (mayores a $0.01 \mathrm{~mm}$ ), es el estado apomórfico (1).

15. Espinas en las camáras medias del atrio genital. La ausencia de espinas en esta región es el caracter plesiomórfico (0), su presencia es el caracter derivado (1).

16. Vagina: La condición plesiomórfica es presentar una vagina (0), mientras que la condición apomórfica (1) es presentar dos vaginas.
17. Vesícula vaginal: La ausencia de una vesícula vaginal contigua a la vagina, es la condición plesiomórfica (0), la presencia de ésta estructura, es la condición apomórfica (1).

18. Número de filamentos del huevo. La presencia de un largo filamento en el polo posterior del huevo, es el carácter plesiomórfico (0), mientras que presentar dos filamentos, es el caracter derivado (1).

\section{RESULTADOS}

El análisis filogenético realizado entre las siete especies de Cynoscionicola, produjo dos cladogramas igualmente parsimoniosos (Figs. 4 y 5) con 35 pasos y un índice de coherencia (consistency index) de 0.85 . En ambos cladogramas se postula a Microcotyle donavini como el grupo hermano del género Cynoscioni-

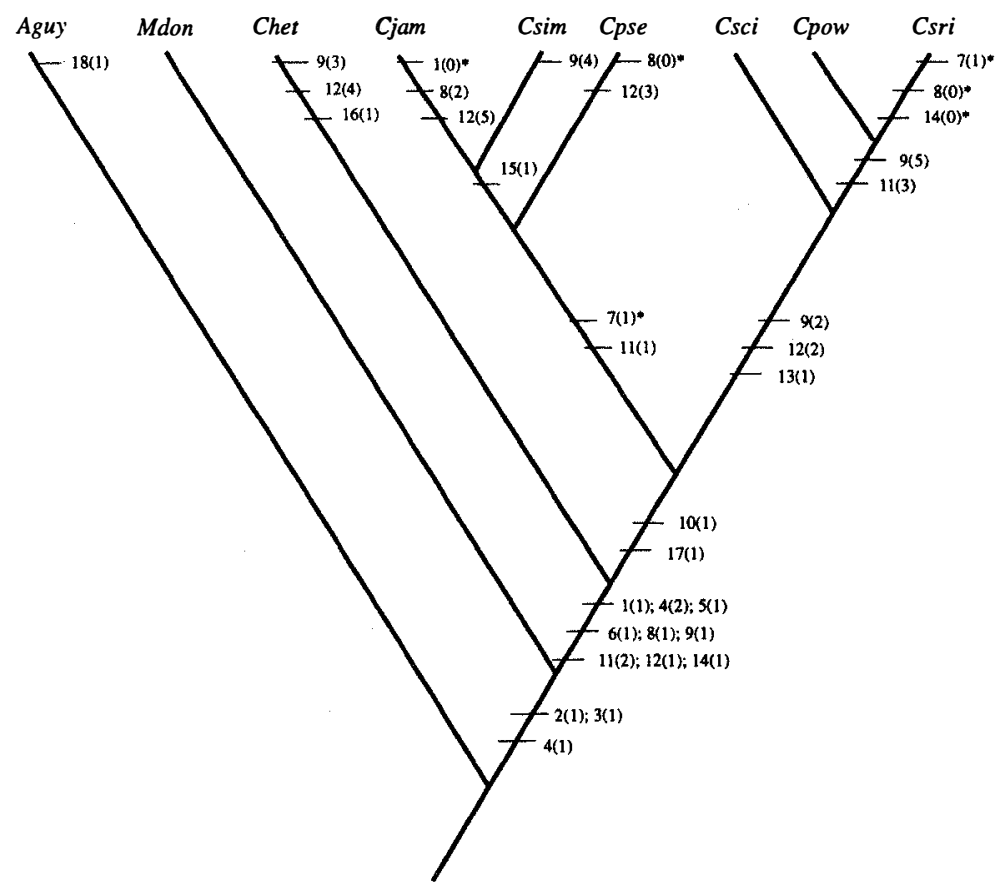

Fig. 4. Cladograma que representa las relaciones filogenéticas entre las especies de Cynoscionicola. Los caracteres marcados con * representan homoplasias. Mdon= Microcotyle donavini; Aguy= Anchoromicrocotyle guaymensis; Chet $=$ Cynoscionicola heteracantha; $C$ pse = C. pseudoheteracantha; $C s r i=C$. srivastavai; $C s c i=C$. sciaenae; Csim= C. similis; Cjam $=$ C. jamaicensis; Cpow $=$ C. powersi. 


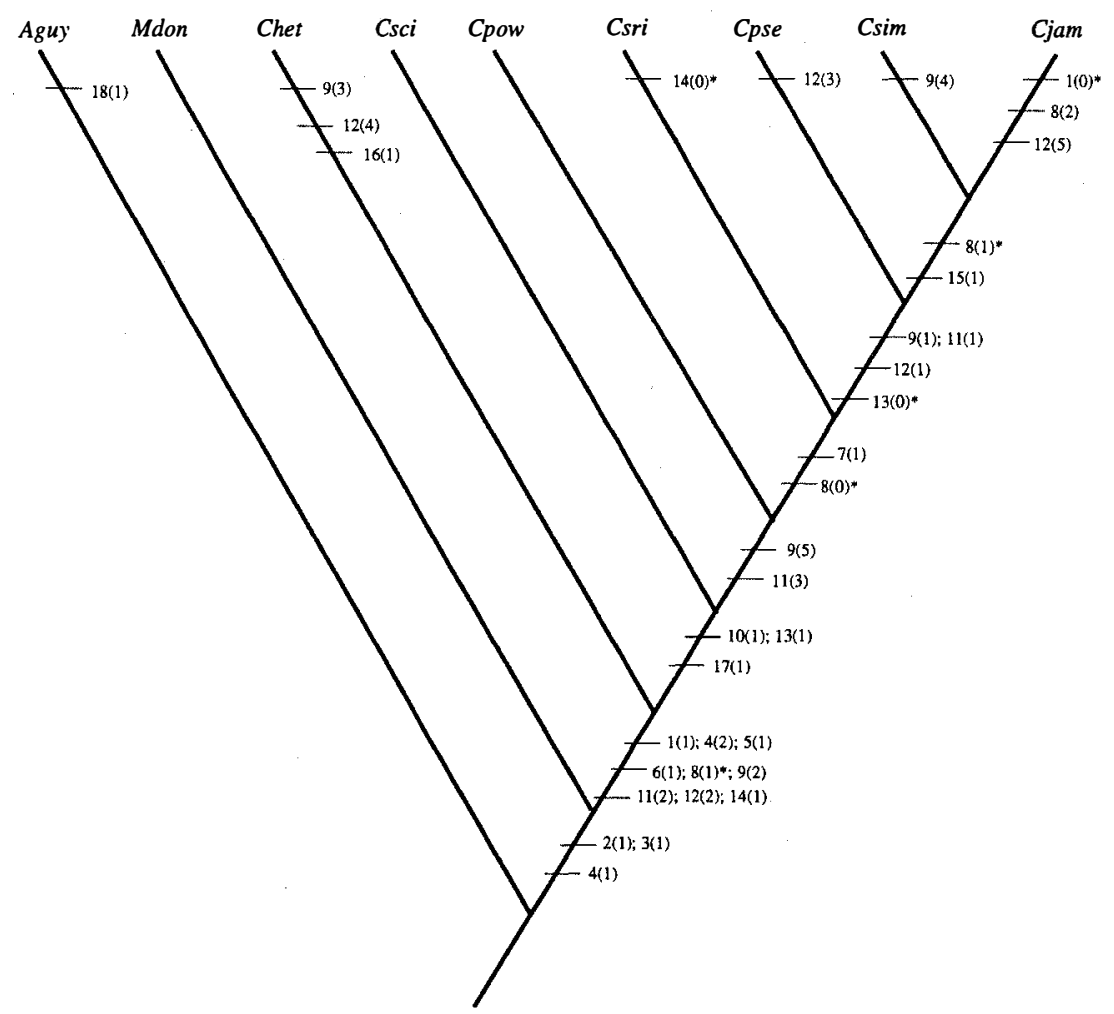

Fig. 5. Cladograma que representa las relaciones filogenéticas entre las especies de Cynoscionicola. Los caracteres marcados con * representan homoplasias. Mdon= Microcotyle donavini; Aguy=Anchoromicrocotyle guaymensis; Chet= Cynoscionicola heteracantha; $C p s e=C$. pseudoheteracantha; $C s r i=C$, srivastavai; $C s c i=C$. sciaenae; $C$ sim $=C$. similis; Cjam = C. jamaicensis; Cpow $=$ C. powersi.

cola y, a Anchoromicrocotyle guaymensis, como grupo hermano de los anteriores. Las diferencias entre los cladogramas son debidas a la posición de un grupo de especies $C$. sciaenae, C. srivastavai $+C$. powersi formando un clado independiente apoyado por tres sinapomorfías: forma de las espinas anterolaterales, forma de las espinas posteriores y número de las espinas posteriores, siendo los dos últimos caracteres no ambiguos, o bien, la posición de estas tres especies como miembros basales de un grupo que incluye a estas tres especies y a $C$. pseudoheteracantha, $C$. similis $+C$. jamaicensis. La posición de $C$. heteracantha como miembro basal del grupo se mantiene en ambos cladogramas.
La monofilia de las especies del género se encuentra apoyada por nueve sinapomorfías que fueron identificadas en este estudio: Ausencia de lengüeta larval, atrio genital constituido por cuatro bolsas musculares, atrio genital armado con espinas de diferente forma y tamaño, forma y tamaño de las espinas anteriores del atrio genital, forma y tamaño de las espinas anterolaterales, forma y tamaño de las espinas posteriores. Tres de éstas (atrio genital constituido por cuatro bolsas musculares, atrio genital armado con espinas de diferente forma y tamaño y forma de las espinas anteriores del atrio genital, con guarda y manubrio evidentes) son caracteres no ambiguos, dado que no 
presentan otros estadios apomórficos en la serie de transformacion como en 9(2), 11(2) y 12(2) y tampoco son caracteres homoplásicos como en $1(0), 8(1)$ y $14(1)$.

\section{DISCUSION}

El resultado del análisis filogenético que aqui presentamos indica claramente que, por un lado, el género Cynoscionicola es un grupo monofilético, estando la monofilia apoyada por tres sinapomorfías no ambiguas. Por otro lado, las relaciones con respecto al grupo externo indican la inestabilidad en la clasificación de los géneros que componen a la familia Microcotylidae. Bravo (1981 b) postuló que el género Cynoscionicola era el único representante de la subfamilia que la autora erigió como Cynoscionicolinae. Más adelante, Mamaev (1986), no reconoce la validez de la subfamilia Cynoscionicolinae e incluye al género Cynoscionicola, junto con el género $A n$ choromicrocotyle, dentro de la subfamilia Anchoromicrocotylinae, erigida por Bravo (1981a), considerando caracteres tales como la presencia de un órgano copulador complejo y una lengüeta larvaria en estado adulto. La clasificación propuesta por Mamaev (1986) fue utilizada inicialmente en este trabajo para establecer las relaciones del grupo externo, considerando a Anchoromicrocotyle como grupo hermano de Cynoscionicola. La utilización de un segundo grupo externo (Microcotyle donavini) contribuyó a demostrar que una vez realizado el análisis filogenético, la propuesta de Mamaev (1986) es errónea y que, ambos géneros, no pueden ser considerados como grupos hermanos y que de hecho no forman parte de la misma subfamilia. Es necesario un análisis filogenético para resolver las relaciones entre los géneros que componen a la subfamilia Microcotylidae, lo cual permitira ratificar la idea de Boeger y Kritsky (1993) de que esta familia constituye un grupo artificial.
El índice de coherencia que se obtuvo para el cladograma que hipotetiza las relaciones filogenéticas entre las especies del género $C y$ noscionicola, es congruente con el obtenido en otros análisis filogenéticos de monogéneos a distintos niveles taxonómicos, desde especies de un género (Measures et al. 1990, Klassen 1994 a,b), hasta relaciones a nivel de familia (Klassen \& Beverly-Burton 1988, Boeger \& Kritsky 1989), lo que indica que es un grupo evolutivamente conservado.

Por otra parte, Brooks \& McLennan (1993 a,b) presentaron evidencias de que, en general, los platelmintos parásitos no exhiben un alto grado de pérdida evolutiva de caracteres y de homoplasia, en comparación con sus grupos hermanos de vida libre, lo que pudiera indicar que estos organismos tienen niveles inusuales de simplificación y/o plasticidad adaptativa; con respecto a las pérdidas evolutivas, considerando la base de datos general para los monogéneos, encontraron que 73 de los 587 caracteres fueron pérdidas, representando el $12 \%$. En nuestro estudio encontramos cuatro perdidas de carácter en ambos cladogramas (pérdida de la lengüeta larval, tamaño de las espinas anteriores, tamaño de las espinas posteriores y número de espinas posteriores) en 35 estados apomórficos lo cual arroja un $11 \%$ de perdida evolutiva de caracteres y coincide totalmente con lo encontrado por Brooks \& McLennan (1993 a,b). Lo anterior indica que las especies del género Cynoscionicola exhiben una mínima cantidad de simplificaciones secundarias en su evolución y más bien, presentan un alto grado de modificación, asociado principalmente a caracteres reproductores (composición del atrio genital).

En la Fig. 6 se representa la optimización tanto de los hospederos como de las áreas geográficas que ocupan, sobre los cladogramas que hipotetizan las relaciones filogenéticas entre las especies de Cynoscionicola. En ambos casos se postula que estos monogéneos son primitiva y predominantemente parásitos de 

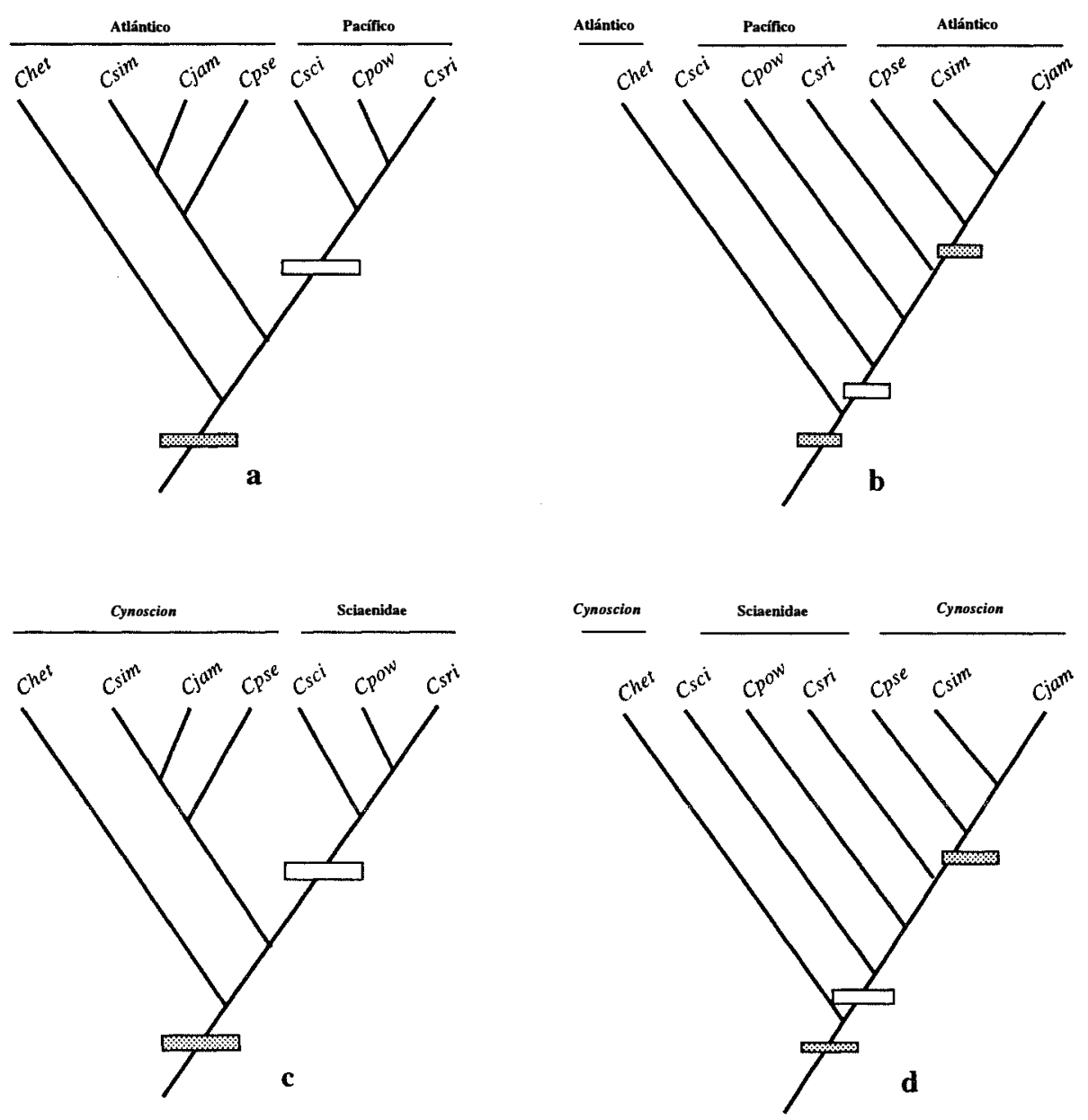

Fig. 6. Cladograma que hipotetiza la historia evolutiva del género Cynoscionicola sobre la cual se optimizó tanto la región geográfica $(\mathrm{a}, \mathrm{b})$, como los hospederos a los que parasita $(\mathrm{c}, \mathrm{d})$. Ver localidades y hospederos en Cuadro 1 . Chet= Cynoscionicola heteracantha; $C p s e=C$. pseudoheteracantha; $C s r i=C$. srivastavai; $C s c i=C$. sciaenae; $C$ sim $=C$. similis; Cjam $=$ C. jamaicensis; Cpow $=$ C. powersi.

peces del género Cynoscion, con un origen en el Atlántico Americano.

Tomando en consideración lo anterior, encontramos que la hipótesis en donde se presentan dos clados bien definidos (Fig 6 a,c), es la más robusta en términos de la explicación que obtenemos al analizar la biogeografía y posible coevolución con los hospederos a los que parasitan. En esta hipótesis se plantea un origen Atlántico en peces del género Cynoscion, con el grupo más derivado parasitando Sciánidos en el Pacífico Americano. Esta hipótesis requiere de dos pasos en cada caso (ver Fig 6 a,c).

Por otra parte, la hipótesis alternativa (Fig. $6 \mathrm{~b}$,d) requiere postular el mismo origen para el grupo, un evento de colonización en Sciánidos del Pacífico y una "reversión" al estado plesiomórfico en el clado más derivado, que incluye a $C$. pseudoheteracantha, C. similis + C. jamaisensis. 
El concepto de especies "gemelas" (geminate species), en combinación con eventos geológicos relativamente recientes, provee apoyo adicional a la primera hipótesis, al analizar el escenario que determinó el proceso de especiación que separó al ancestro del "clado del Pacífiço" (C. sciaenae, C. srivastavai $+C$. powersi) del clado del Atlántico ( $C$. pseudoheteracantha, C. similis $+C$. jamaisensis). Las especies "gemelas" son aquellas especies hermanas que aparecen en lados opuestos de alguna barrera geográfica; este concepto fue aplicado recientemente por Marques et al. (1996) al caso de los organismos parásitos, en especies de tetrafilídeos (Cestoda) parásitos de mantarrayas que están separados, en las costas del Atlántico y del Pacífico, por el Istmo de Panamá, mismo que surgió como un puente de conexión entre Norte y Sudamérica recientemente (3.5-5.7 millones de años según Lessios 1979).

De acuerdo con lo anterior, el ancestro del "clado Pacífico" y del "clado Atlántico", tenian una distribución en ambos litorales y el evento de especiación que condujo a la separación de ambos linajes fue producto del surgimiento del Istmo de Panamá en el Plioceno, junto con una distribución secundaria, colonización o cambio de hospedero (host-switching) a otros Sciánidos. Este patrón de especiación es alopátrico por separación geográfica (vicarianza) de acuerdo con Brooks y McLennan (1991) y produjo asi dos especies "gemelas", representadas por los ancestros hipotéti$\cos$ que posteriormente dieron origen, por un lado, a las especies $C$. similis $+C$. jamaisensis y $C$. pseudoheteracantha (Atlántico), mientras que por el otro, al clado formado por $C$. sciaenae y $C$. srivastavai $+C$. powersi (Pacífico) Adicionalmente al aislamiento geográfico el "clado Pacífico" tuvo un evento de colonización a otros miembros de la familia Sciaenidae, tales como Umbrina, Bairdiella, Isopisthus y Sciaena. El surgimiento del Istmo de Panamá como un factor que condujo a eventos de especiación y posible formación de especies "gemelas", tambien fue descrito recientemente para otro grupo de monogéneos de la familia
Pterinotrematidae por Pérez-Ponce de León $e t$ al. (1997).

El origen Atlántico, en peces del género Cynoscion para las especies de Cynoscionicola, es congruente con la hipótesis de relaciones filogenéticas para los miembros de Cynoscion presentada por Sasaki (1989). Este autor determinó que la distribución actual de éstos es exclusiva del nuevo mundo y es Anfiamericana es decir, en ambas costas del Continente Americano; adicionalmente, las especies del género tienen rangos de distribución muy restringidos (Nelson, 1994). Sasaki (1989) postuló que el ancestro del clado formado por Cynoscion + Macrodon + Isopisthus + Plagioscion apareció en el Atlántico Sudamericano. Nuestros resultados apoyan esta propuesta ya que el miembro basal $C$. heteracantha tiene una amplia distribución en las Costas del Atlántico particularmente en E.U., desde Florida hasta Masachusets.

\section{AGRADECIMIENTOS}

Agradecemos a Virginia León Règagnon y Luis Garcia Prieto por la revisión y comentarios realizados al manuscrito. A Ralph Lichtenfels, curador del U. S. National Para ite Collection, Beltsville, Maryland y Scott Gardner, curador de la UNSMHWML por el envío de holotipos y paratipos, depositados en estas coleciones y Agustín Jiménez Ruiz, por la ayuda proporcionada en la revisión del material.

\section{RESUMEN}

En este trabajo se presenta un análisis filogenético para siete-especies nominales del género Cynoscionicola Price 1962, el cual esta basado en 18 caracteres y 31 estadós de carácter. El análisis produjo dos cladogramas igualmente parsimoniosos con un índice de coherencia de 0.85 y 35 pasos. Las diferentes topologías de los cladogramas dependen de la posición de los miembros que habitan en Scíanidos de la costa del Pacífico, con respecto a aquellas que habitan en Cynoscion en la costa del Atlántico. La 
monofilia de Cynoscionicola es apoyada por tres sinapomorfías no ambigüas: atrio genital formado por cuatro bolsas atriales, atrio genital armado con espinas de distinta forma y tamaño y forma de las espinas anteriores del atrio genital. Usando el análisis filogenético como un "templado", analizamos las relaciones con el hospedero y la distribución geográfica de los miembros de Cynoscionicola, proponemos un origen para ambos, hospederos y monogéneos, en el Atlántico, con una ruta de dispersión temprana a lo largo de la costa del Atlántico, un evento de especiación por vicarianza, determinado por el aislamiento de una población de Cynoscion cuando surgió el Istmo de Panamá en el Plioceno, en combinación con un evento de colonización a otros miembros de la familia Scianidae.

\section{REFERENCIAS}

Bravo, H. M. 1981a. Helmintos de peces del Pacífico Mexicano XXXVI. Sobre un género y subfamilia nuevos de la famulia Microcotylidae Taschemberg, 1879 emend. An. Inst. Cienc. Mar y Limnol., UNAM. 8: 305-314.

Bravo, H. M. 1981b. Helmintos de peces del Pacífico Mexicano XXXVIII. Estudio de monogéneos del suborden Microcotylinea Lebedev, 1972, con la presentación de una subfamilia y una especie nuevas. An. Inst. Biol., UNAM. 52: 13-26

Boeger, W. A. \& D. C. Kritsky. 1989. Phylogeny, coevolution and revision of the Hexabothriidae Price, 1942 (Monogenea). Int. J. Parasitol. 19: 425-440.

Boeger, W. A. \& D. C. Kritsky. 1993. Phylogeny and a revised classification of the Monogenoidea Bychowsky, 1937 (Platyhelmintes). Syst. Parasitol. 26 : 1-32

Brooks, D. R. \& D. A. McLennan. 1991. Phylogeny, Ecology and Behaviour. A Research Program in Comparative Biology. University of Chicago, Chicago,. Illinois 434p.

Brooks, D. R. \& D. A. McLennan. 1993a. Parascript: Parasites and the lenguage of evolution. Smithsonian Institution, Washington, D. C. 429 p.

Brooks, D. R. \& D. A. McLennan. 1993b. Macroevolutionary trends in the morphological diversification among the parasitic flatworms (Platyhelmintes: Cercomeria). Evolution 47: 495-509.

Jiménez R., A. 1996. Taxonomía y descripción de las comunidades de helmintos parásitos de Umbrina xanti Gill,
1862 (Pisces: Scianidae) en la Bahía de Chamela, Jalisco, México. Tesis de Licenciatura. UNAM. México.

Klassen, G. J. 1994a. Phylogeny of Haliotrema species (Monogenea: Ancyrocephalidae) from Boxfishes (Tetraodontiformes: Ostraciidae): Are Haliotrema Species from Boxfishes Monophyletic?. J. Parasitol. 80: 596-610.

Klassen, G. J. 1994b. On the monophyly of Haliotrema species (Monogenea: Ancyrocephalidae) from Boxfishes (Tetraodontiformes: Ostraciidae): Relationships within the Bodiani group. J. Parasitol. 80: 611-619.

Klassen, G. J. \& M. Beverly-Burton. 1988. North American freshwater Ancyrocephalids (Monogenea) with articulating haptoral bars: Phylogeny reconstruction. Syst. Parasitol. 11: 49-57.

Lambert, M. \& L. Euzet. 1979. Espéces nouvelles du genre Cynoscionicola Price, 1962 (Monogenea: Microcotylidae). Z. Parasitenk. 60: 229-237.

Lessios, H. A. 1979 Use of Panamanian sea urchins to test the molecular clock. Nature 280: 599-561.

Maddison, P., M. Donaghue \& D. Maddison. 1984. Outgroup analysis and parsimony. Syst. Zool. 33: 83-103.

Mamaev, Yu. L. 1986. The taxonomical composition of the Family Microcotylidae Taschenberg, 1879 (Monogenea). Folia Parasitologica. 33: 199-206.

Marques, F., D. Brooks \& H. Molina. 1996. Two new species of Tetraphyllidean Cestodes in Himantura pacifica (Chondrichthyes: Myliobatiformes: Dasyatididae) from the Northwest Coast of Costa Rica. J. Parasitol. 82: 302-306.

Measures, N., M. Beverly-Burton \& A. Williams. 1990. Three new species of Monocotyle (Monogenea: Monocotylidae) from the stingray, Himantura varnak (Rajiformes: Dasyatidae) from the Great Barrier Reef: Phylogenetic, reconstruction, systematics and emended diagnoses. Int. J. Parasitol. 20: 755-767.

Nelson, J. 1994. Fishes of the World. Wiley, Nueva York. $600 \mathrm{p}$.

Pérez-Ponce de León, G., V. León-Regagnon \& B. Mendoza-Garfias. 1997. Análisis filogenético de la familia Pterinotrematidae Bychowsky y Nagibina, 1959 (Platyhelminthes: Moonogenea). An. Inst. Biol. UNAM. 68: (en prensa)

Sasaki, K. 1989. Phylogeny of the Family Sciaenidae, with notes on its Zoogeography (Teleostei: Perciformes). Mem. Fac. Fish Hokkaid Univ. 36: 1-137. 
Sasaki, K. 1989. Phylogeny of the Family Sciaenidae, with notes on its Zoogeography (Teleostei: Perciformes). Mem. Fac. Fish Hokkaid Univ. 36: 1-137.

Swofford, D. L. 1993. Phylogenetic analysis using parsimony. Version 3.1.1. Illinois Natural History Survey, Urbana, Illinois.
Watrous, E. \& Q.Wheeler. 1981. The out-group comparasion method of character analysis. Syst. Zool. 30: 1-11.

Wiley, E. O. 1981. Phylogenetics. The theory and practice of phylogenetic systematics. Wiley, Nueva York. $439 \mathrm{p}$ 